\title{
DIE AMERIKANISCHE SCHWERINDUSTRIE
}

\author{
Von HaNs BoEsch
}

Mit 1 Karte

Die Eisen- und Stahlindustrie ist ohne Zweifel die Basisindustrie unseres technischen Zeitalters. Direkt oder indirekt reichen die Wurzeln fast aller technischen Wirtschaftszweige in die Schwerindustrie hinein. Reichtum an Basisrohstoffen, die Existenz der zur Erzeugung von Eisen und Stahl notwendigen Einrichtungen und Wirtschaftsorganisationen und nicht zuletzt ein stimulierender Markt vermögen in ihrer Gesamtheit schon in erster Annäherung das Wirtschaftspotential eines Landes zu charakterisieren und außerdem die Gravitationspunkte im Bilde der Wirtschaftslandschaften der Erde aufzudecken.

Die USA erzeugten vor dem zweiten Weltkriege schon über ein Drittel des auf der ganzen Erde hergestellten Stahles; heute dürften es rund $50 \%$ sein, womit die USA zum absoluten Schwerpunkt der Eisen- und Stahlerzeugung wurden. Tabelle 1 enthält die grundlegenden Zahlen zur Illustration dieser Entwicklung, die besonders imposant ist, wenn wir damit den nur etwa $7 \%$ betragenden Anteil an der Weltbevölkerung vergleichen.

Tab. 1: US-Erzeugung in \% am Welttotal

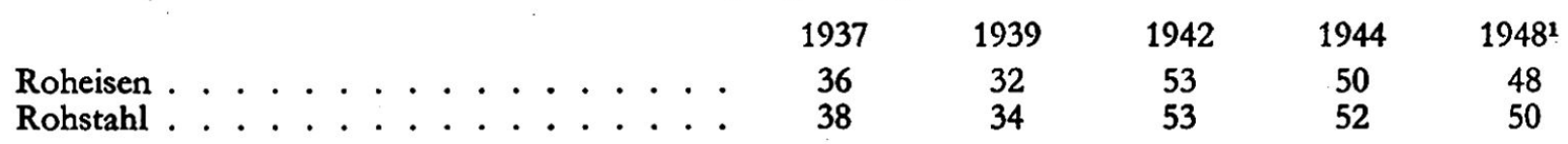

Die folgenden Ausführungen sollen, ausgehend vom Bilde der Vorkriegsstruktur, die Veränderungen der amerikanischen Schwerindustrie während des Krieges zeigen und einige Hinweise auf die mögliche Entwicklung geben. Damit dies in knapper Form geschehen kann, müssen einige Erläuterungen allgemeiner Natur vorangestellt werden.

Die Eisen- und Stahlindustrie baut sich aus folgenden vier Arbeitsphasen auf: 1. In den Bergbaugebieten erfolgt der Abbau der eisenhaltigen Erze; 2. In den Verhüttungsanlagen (meist in Hochöfen) wird durch Schmelzen und Reduzieren der meist oxydischen Erze unter Abgang von Gasen und Schlacke das Roheisen gewonnen; 3. Im Stahlwerk werden in einem oft recht komplexen Arbeitsverfahren dem Roheisen durch Entzug und Beimischung. von bestimmten Stoffen jene Eigenschaften gegeben (z. B. Schmiedbarkeit, Härte usw.), die ihm die Bezeichnung Stahl verleihen; 4. Im Walzwerk wird aus den glühenden Stahlblöcken eine ganze Reihe von Erzeugnissen geschaffen, die teilweise, wie Eisenbahnschienen u. a., direkt Verwendung finden, meistenteils jedoch in weiteren Arbeitsgängen erst ihrer endgültigen Bestimmung zugeführt werden. Bestimmte Materialmengen passieren jedoch nicht alle vier der genannten Arbeitsphasen, sondern werden - sei es als Roheisen oder Stahl - zu Stücken gegossen, die ebenfalls teilweise direkt Verwendung finden können, meistens jedoch weitere hier nicht zu untersuchende Arbeitsgänge durchlaufen².

Ursprünglich mußte alles Eisen primär aus Eisenerz, aller Stahl aus Roheisen gewonnen werden. Im Laufe der Entwicklung stand aber in zunehmendem Maße Altmaterial, Schrott, zur Verfügung, sei es, da $\beta$ im Laufe des Verarbeitungsprozesses solches Material anfiel oder da $\beta$ es als eigentliches Alteisen nach Erfüllung seiner Zweckbestimmung in den Arbeitsprozeß zurückkehrte. Dieser Schrott der Amerikaner unterscheidet zwischen sogenanntem home und market scrap - kehrt im Kreislauf in den oben dargestellten Arbeitsgang zurück, und zwar in erster Linie in die Stahlwerke, während es in der Beschickung der Hochöfen nur eine kleine Rolle spielt.

Nachdem sich seit Jahrzehnten das Schwergewicht innerhalb der Eisen- und Stahlindustrie auf die Stahlwerke verlagert hatte, begann sich dort der wachsende Schrottanfall auch auf die technische Gestaltung des Arbeitsvorganges auszuwirken. In Amerika wird nur ein kleiner Teil (1945 etwas mehr

1 Schätzung.

${ }^{2}$ Die sehr wichtige Frage der Versorgung der amerikanischen Schwerindustrie mit Mangan und Stahlveredlern wird hier nicht untersucht. 
als $6 \%$ ) nach dem Bessemerverfahren, die überwiegende Mehrheit des Stahles (88\%) nach dem Openhearth-Verfahren, der Rest im Elektrostahlofen hergestellt. Das Open-hearth-Verfahren benötigt große Schrottmengen zur Stahlherstellung, im Jahre 1938 51,5\% gegenüber 48,5\% Roheisen; beim Bessemerverfahren werden dagegen nur 5-6\% Schrott, beim Elektrostahlofen jedoch über $98 \%$ benötigt. Die kriegsbedingte Produktionssteigerung verlangte deshalb auch einen starken Anstieg der Schrottlieferungen, die zum Teil durch fast völlige Unterbindung der früher bedeutenden Schrottausfuhren erreicht wurden. Außerdem wurde der Schrottanteil auf 46,5\% (1942) heruntergedrückt und durch eine stark gesteigerte Roheisenerzeugung ein gewisser Ausgleich geschaffen. Diese Ausführungen illustrieren das feine Ineinanderspielen der einzelnen Faktoren.

Im folgenden werden uns technische Aspekte jedoch nur so weit beschäftigen, als sie von Einfluß auf die Standorte der Schwerindustrie sind. Denn uns interessieren in erster Linie jene Fragen, die mit der Standortswahl zu tun haben. Um die konkreten Verhältnisse besser zu verstehen, erscheint es nützlich, hier vorerst einige allgemeine Überlegungen anzustellen.

Innerhalb derjenigen Verarbeitungsphasen, die wir hier betrachten, tragen alle Produkte (nämlich Eisenerz, Roheisen, Rohstahl, Walzwerkerzeugnisse, Eisen- und Stahlschrott wie Koks und Zuschläge) den Charakter von billigen Massengütern. Während der geringe spezifische Wert längeren Transporten entgegensteht, vermögen dem Transportvolumen entsprechende besondere Transporteinrichtungen diesen Nachteil weitgehend wieder auszugleichen. Dabei spielt die Geeignetheit des Gutes für den Transport und den Umlad eine große Rolle.

Die Vermeidung längerer Transporte führt an sich schon zur räumlichen Ballung der einzelnen Arbeitsphasen. Diese Integration wird auch aus energiewirtschaftlichen Gründen angestrebt: das noch flüssige Roheisen wird direkt ins Stahlwerk, die glühenden Rohstahlblöcke ins Walzwerk übergeführt. Eine solche durchgreifende Integration ist aber nur dort denkbar, wo sehr große Mengen in gleichbleibenden Qualitäten erzeugt werden. Damit nimmt die Möglichkeit zur Integration bei fortschreitendem Arbeitsprozeß und steigender Differenzierung ständig ab. Die amerikanische Eisen- und Stahlindustrie trägt, wie wir noch zeigen werden, den Stempel dieser Gegebenheiten nicht nur in ihrer räumlichen Verteilung, sondern auch in der wirtschaftlichen Struktur und unterscheidet sich damit grundsätzlich von anderen großen Industriezweigen, z. B. der Textilindustrie.

Von großer Bedeutung erscheint nach unseren Ausführungen über die technischen Fragen zweifellos auch der Zeitfaktor. Während der letzten 150 Jahre wurde Roheisen fast ausschließlich im sogenannten Blashochofen erzeugt, in welchem Koks besonderer Qualität als Reduktionsmittel und zur Erzeugung der Schmelzwärme zur Verwendung kommt. Damit bestimmten in der ersten Entwicklungsphase die damals gültigen Standortsbedingungen des Blashochofens weitgehend den Standort der gesamten Schwerindustrie. In dem Maße jedoch, wie sich die Schwerpunktsverlagerung zur Stahlerzeugung vollzog, traten neue, komplexere Standortsbedingungen hinzu, wobei nun vor allem die Marktorientierung wichtig wurde. Ganz allgemein gilt für Schwerindustriezentren auf der Basis des Blashochofens - die im Prinzip die Masse der amerikanischen ausmachen -, daß sie sich entweder an oder in nächster Nähe der Kohlenbecken, die den metallurgischen Koks liefern; finden oder dann doch durch bestimmte funktionelle Beziehungen mit diesen verbunden sind. In einer großen Überschau darf aber nicht übersehen werden, daß frühere Verhüttungsverfahren zu einer ganz andern Standortswahl führten - es sei nur an den einst weltbeherrschenden, sehr dispers struierten mittelschwedischen Bergslagendistrikt erinnert, oder daran, daß heute erneut moderne Verhüttungsverfahren, wie der elektrische Niederschachtofen, ganz andere Standortsbedingungen aufweisen.

Die heute im Durchschnitt benötigten Rohstoffmengen im Verhüttungsprozeß scheinen der Grundregel, daß "das Erz zur Kohle geht», zu widersprechen: 1944 betrug je Tonne ${ }^{3}$ Roheisen der Einschlag 1,892 Tonnen eisenhaltiges Material, davon 1,725 Tonnen Erz und 0,045 Tonne Schrott, gegenüber nur 0,905 Tonne Koks und 0,389 Tonne Zuschlag in Form von Kalken und Dolomiten. Zudem handelt es sich bei Koks um ein Gut mit höherem spezifischem Wert als bei Eisenerz. Wenn wir in den Vereinigten Staaten gleich wie in Europa die Schwerindustrie in erster Linie an der Koksbasis finden, dann müssen offenbar andere Gründe als die heute gültigen Rohstoffmengen und -preise dabei eine Rolle spielen. Auf die größere Eignung von Eisenerz zum Massentransport wurde schon hingewiesen; zu erwähnen wäre auch, daß in vielen Fällen verschiedene Lieferanten an der Beschickung eines Hochofens mit Erz beteiligt sind, während bei Koks nur einer in Frage kommt. Vor allem aber gilt es zu beachten, daß das angegebene Gewichts- wie das Preisverhältnis Erz zu Koks früher wesentlich vom heutigen verschieden war. Erst ein langer technischer Entwicklungsprozeß vermochte den Koksanteil auf den heutigen Stand hinunterzudrücken. Da gleichzeitig die relativen Kokspreise früher niedriger waren, übte die Kohlenbasis in der Zeit der ersten kräftigen schwerindustriellen Entwicklung stärkste attraktive Wirkung aus. Fast ausnahmslos ging damals, wie man zu sagen pflegt, das Erz zur Kohle. Die vorstehend erwähnten Änderungen befreiten die Industrie von dieser engen Bindung und leiteten einen wichtigen Dezentralisationsprozeß ein. Retardierend wirken dabei aber vor allem die folgenden drei Momente: erstens übt die Kohlenbasis über die an sie gebundenen weiteren Industrien, die für die Schwerindustrie Markt und Schrottlieferant sind, indirekt wirkende Anziehung auf die Schwerindustrie

${ }^{3}$ Hier wie im folgenden wird überall mit sogenannten «short tons» zu 907,18 kg gerechnet, wie dies in Amerika üblich ist. 
aus; zweitens besitzt jede Industrieanlage auch bei Benachteiligung durch veränderte Umweltfaktoren ein bestimmtes Beharrungsvermögen; und drittens wird dieses inherente Beharrungsvermögen durch die interessierten Kreise mit den Mitteln der Preis-, Frachttarif- und Zolltarifgestaltung in vielen Fällen noch vertieft.

Nach diesen einleitenden technischen und wirtschaftlichen Bemerkungen lassen sich die einzelnen Schwerindustriezentren der USA fast zwanglos in den Rahmen der in Frage kommenden Gegebenheiten einfügen.

Seit etwa in der zweiten Hälfte des 19. Jahrhunderts begann die amerikanische Schwerindustrie an der pennsylvanischen Kohlenbasis mit dem Zentrum Pittsburgh rasch emporzuwachsen. Die Oberläufe des Ohiosystems erschließen in ihren Tälern in Pennsylvania, Westvirginia und Kentucky leicht abbaubare mächtige Kohlenflöze, die vorzüglichen metallurgischen Koks liefern. Qualität des Rohstoffes, Leichtigkeit des Abbaus, im Verein mit dem natürlichen Wasserstraßennetz, machten aus dieser Region den dominierenden Kokslieferanten der USA.

Noch ausgesprochener ist die Konzentrierung des Erzbergbaus im Lake Superior District, der 80 und mehr Prozent der Erzförderung der USA stellt. Die periphere Lage im Verein mit den genannten Faktoren bewirkt, daß praktisch die ganze ungeheure Menge dem Koks zu- oder entgegengeführt wird.

Zwischen diese zwei Pole, zu denen als dritter das große Verbrauchsgebiet des Nordostens und des Mittleren Westens tritt, ist von Anfang bis heute die Masse der amerikanischen Schwerindustrie eingespannt. Dabei spielt Pittsburgh immer noch die Rolle des absoluten Zentrums mit 31\% Roheisen- und 31\% Rohstahlanteil (1943). Eine erste Dezentralisierung erfolgte durch die sinngemäße Ausnützung der leer von Pittsburgh an die Umschlagplätze am Eriesee rollenden Erzzüge durch Koks; die am Südufer mit Zentrum Cleveland emporgewachsene Industrie weist $23 \%$ Roheisen- und $21 \%$ Rohstahlanteil auf. Eine zweite Etappe der Dezentralisierung folgte dem Gefälle zum wichtigsten Verbraucher- und Verteilerplatz an das Südufer des Michigansees (Chicago); der Anteil an der Roheisenerzeugung beträgt hier $23 \%$, an Rohstahl $20 \%$. $3 \%$ Roheisen und $4 \%$ Rohstahl sind schließlich an der Eisenerzbasis am Obern See lokalisiert. Eingespannt in die erwähnten Pole sind demnach rund $80 \%$ der Roheisenund $76 \%$ der Rohstahlerzeugung. Es ist zu beachten, daß diese ganze Gruppe ausgesprochen auf den Binnenmarkt ausgerichtet ist und, abgesehen von der Richtung nach Kanada, eine schlechte Außenhandelslage besitzt. Die geringe Verflechtung mit dem Welthandel ist bis 1939 für die Gesamtheit der amerikanischen Eisen- und Stahlindustrie bezeichnend gewesen.

Mit zirka $14 \%$ Anteil an der Roheisenerzeugung und $15 \%$ Rohstahl (inklusive W. Va.) steht an nächster Stelle der sogenannte Mittelatlantische Distrikt mit Schwerpunkten um Baltimore und New York. Seine Eisenerzbasis liegt teilweise im nähern und weitern Hinterland; in erster Linie werden Erze von Übersee, vor allem aus Chile und Cuba, importiert. Koks wird per Achse aus dem Innern zugeführt. Dieser Distrikt ist der einzige, der eine vorzügliche Import-Export-Lage besitzt.

Mit 6\% Roheisen- und $4 \%$ Rohstahlanteil folgt der in Alabama gelegene Birmingham District. Seine Vorteile bestehen darin, daß sämtliche Rohstoffe sich in unmittelbarer Nähe der Industrie befinden, womit er zum billigsten Roheisenproduzenten wird. Qualitative Mängel der Rohstoffe, die verteuernd wirken, vor allem aber die Lage inmitten einer mehrheitlich agrarwirtschaftlich orientierten Großregion der USA stellen wichtige Nachteile dar. Mit dem Golf ist Birmingham durch eine Wasserstraße verbunden und besitzt damit eine relativ gute Exportlage.

Bis zum zweiten Weltkriege besaß der ganze Westen neben zahlreichen Stahlwerken und einem sekundären Zentrum in Pueblo, Colorado, keine Schwerindustrie von Belang.

Damit wäre das Bild der Verteilung der Schwerindustriezentren vor Ausbruch des zweiten Weltkrieges gezeichnet. Um das Verhalten derselben in den nachfolgenden 


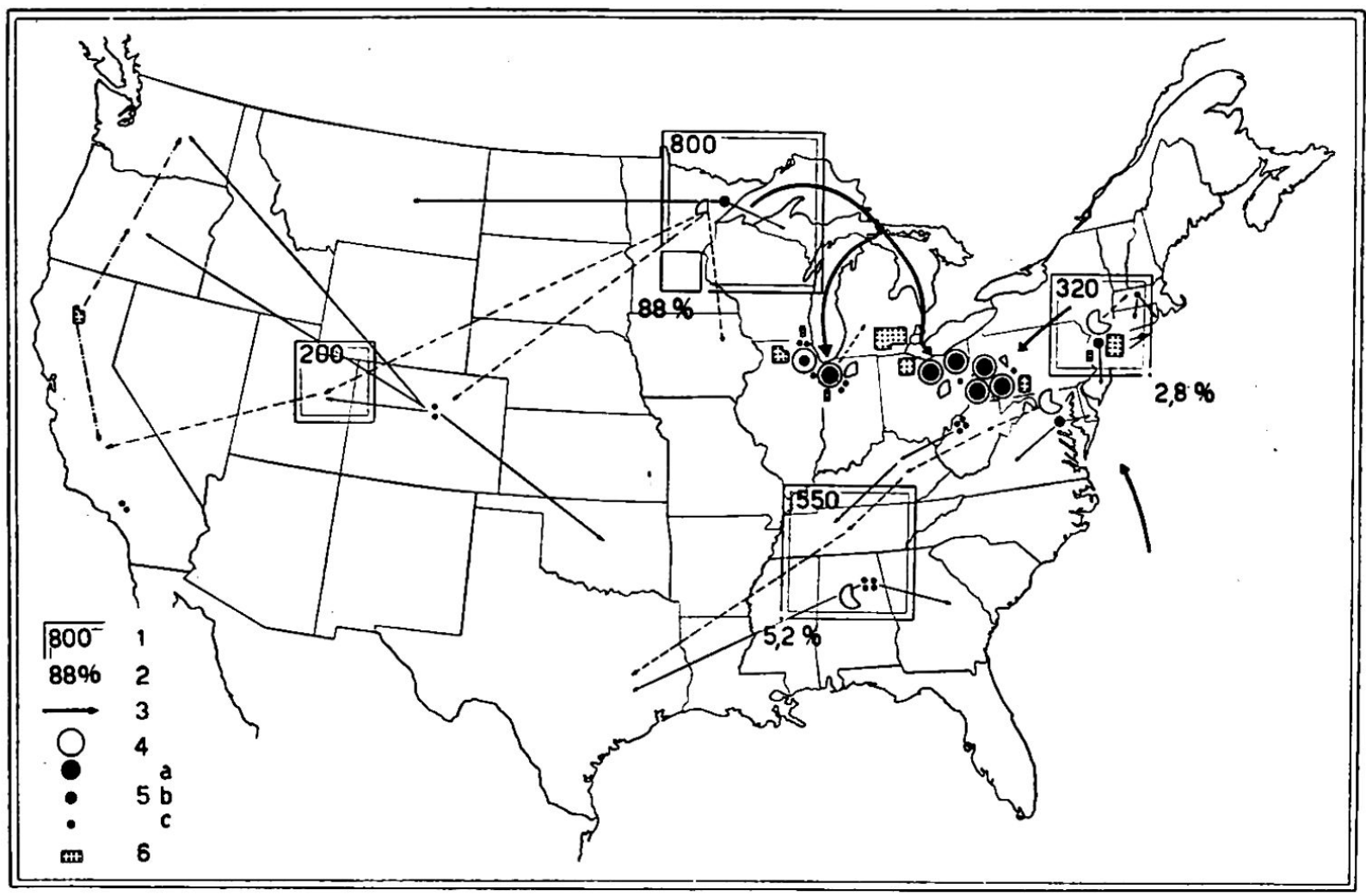

Die amerikanische Schwerindustrie. 1 : Eisenerzreserven (actual reserves) in Millimetertonnen. 2: Anteil an der US-Eisenerzförderung 1943. 3: Hauptbewegungen der Eisenerztransporte. 4: Anteil US-Roheisenerzeugung 1943 (Kreis = 10\%). 5: Anteil an der US-Rohstahlerzeugung 1943 (Kreis a $10 \%$, Kreis b $5 \%$, Kreis c 1\%). 6: Anteil am US-Fertigstahlverbrauch (finished industrial steel) 1935 (1 Punkt $=1 \%$ ). Wo sich die Zahlenangaben auf mehrere Staaten beziehen, ist dies durch verbindende Linien angegeben (gestrichelte Linien für Eisenerzerzeugung, ausgezogene Linien für Rohstahl und strichpunktierte Linien für Angaben über den Stahlverbrauch)

Jahren zu verstehen, sind jedoch noch einige Besonderheiten etwas näher zu untersuchen. Es wurde schon darauf hingewiesen, daß die Eisen- und Stahlindustrie in weit höherem $\mathrm{Maße}$ als andere wichtige Industriezweige die Tendenz zur Integration besitzt. Neben der rein technisch-betrieblichen Integration des Arbeitsprozesses ist dabei auch an die horizontale und vertikale Zusammenfassung in einzelnen großen Gesellschaften zu denken. Dabei nimmt in der Regel bei fortschreitendem Arbeitsgang die Tendenz zur Zusammenschließung ab.

Die bedeutendste dieser integrierten Gesellschaften, die United States Steel Corporation, erzeugte 1940 von der gesamten US-Produktion 40,1\% Roheisen, $34 \%$ Rohstahl und $31,3 \%$ der Walzwerkerzeugnisse; $40 \%$ des gesamten in der amerikanischen Eisenund Stahlindustrie investierten Kapitals entfielen auf sie. Zusammen mit der Bethlehem Steel Corporation und der Republic Steel Corporation lauten die Zahlen: 61,6\% Roheisen, 57,7 \% Rohstahl, 52,6\% Walzwerkerzeugnisse und 63\% des investierten Kapitals. Zusammen mit zehn weiteren großen Gesellschaften steigen diese Werte auf 87,9\% Roheisen, 86,4\% Rohstahl, 80,1\% Walzwerkerzeugnisse und $88 \%$ des investierten Kapitals.

Die Tätigkeit der großen Gesellschaften erstreckt sich jedoch in beiden Richtungen über die oben angegebenen Arbeitsphasen hinaus. Vor allem ist dies in der Kontrolle und der Ausbeutung der Eisenerzlager der Fall. US Steel kontrolliert mehr als die Hälfte der entscheidenden Erzreserven am Lake Superior. Ausschließlich für Lieferungen an die US Steel förderte deren Tochtergesellschaft Oliver Iron Mining Co. 1937 42,2\% der Lake-Superior-Produktion, die auf den Schiffen der Pittsburgh Steamship Co. - 
der größten Dampfergesellschaft auf den Great Lakes - transportiert wurden. Mit 21,9 und $9,1 \%$ folgen erst die nächsten erzfördernden Gesellschaften.

Ein erster Vergleich der oben angeführten Prozentzahlen mit den absoluten Produktionsangaben für Roheisen, Rohstahl usw. zeigt, da 3 die Integration in den ersten Arbeitsphasen am stärksten ist. Daraus folgert, daß die großen Gesellschaften auch als wichtige Verkäufer von Roheisen auf dem offenen Markt in Frage kommen; anderseits ergibt sich, daß nur ein kleiner Teil des Rohstahles auf dem offenen Markt verkauft wird. Der Großteil wird zur weiteren Verarbeitung in den Walzwerken der großen Gesellschaften herangezogen. Erst bei der weiteren Verarbeitung zeigt sich dann ein deutlicher Wechsel: Vor dem zweiten Weltkriege dürfte etwa die Hälfte'des Stahlgusses und der Walzwerkerzeugnisse schon aus dem integrierten Arbeitsprozeß ausgeschieden sein; die andere Hälfte wurde noch einer bestimmten anfänglichen Weiterverarbeitung unterzogen, um dann ebenfalls an selbständige Gesellschaften zur weiteren Verwendung verkauft zu werden. Nur ein relativ kleiner Teil verblieb (vor allem im Schiffsbau, Brücken usw.) den Gesellschaften, die als Tochtergesellschaften der Giganten der Schwerindustrie anzusprechẹn sind. Es wird angenommen, daß während und nach dem zweiten Weltkriege die integrierten Gesellschaften stärker auch in dieser Richtung vorgedrungen sind, doch fehlen darüber statistische Angaben. Die folgende tabellarische Zusammenstellung (Tab. 2) zeigt besser als längere Ausführungen, in welchen Industrien und in welchen Gebieten dieser sogenannte "finished industrial steel » abgesetzt wurde. Eisenbahnschienen, Röhren für Pipelines und zahlreiche Konstruktionsstähle, die ohne weitere Verarbeitung Verwendung finden, blieben dabei unberücksichtigt. Gleichzeitig charakterisiert diese Tabelle die einzelnen Verbrauchsgebiete auf das beste.

Tab.2: Absatzgebiete und Bezüger von sogenanntem "finished industrial steel» 1935

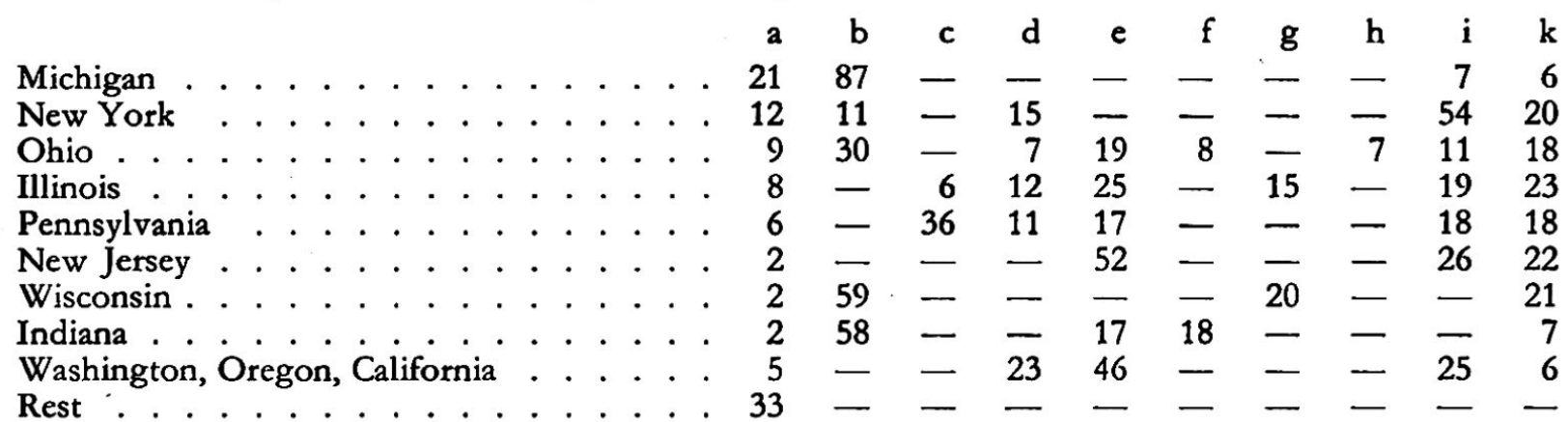

Erläuterung: a): \% der US; b) bis k) in \% des Staatstotals: b). Automobile, c) Eisenbahnwagen, d) Bauten, e) containers, f) elektrische Ausrüstung, g) landwirtschaftliche Maschinen, h) Büroeinrichtungen, i) Makler und Großhandel, k) Rest.

Diese Ausführungen über die gesellschaftliche Integration innerhalb der amerikanischen Schwerindustrie waren im Rahmen unserer Ausführungen von großer Wichtigkeit. Bei der Diskussion von Standortsfragen macht man nämlich immer wieder die Beobachtung, daß die einzelnen Industrien gleichsam wie willenlose Gestalten in einem Magnetfelde betrachtet werden, die ohne weiteres dem sogenannten "optimalen" Standorte zustreben. Ihre einzelnen Teile ordnen sich aber keineswegs unabhängig voneinander räumlich ein, sondern auch in einem privatwirtschaftlich organisierten Staate verhalten sie sich unter den aufgedeckten Verhältnissen nach dem planenden Willen einiger weniger Gesellschaftsleitungen. Damit finden wir die einzelnen Arbeitsphasen oft nicht mehr dort lokalisiert, wo sie sich bei vollkommener Desintegration finden würden, sondern sie haben als Einzelteile einen im ganzen sinnvollen, wenn auch, für sich betrachtet, häufig nicht optimalen Standort einzunehmen. Von den drei erwähnten Mitteln, die dabei angewendet werden können und die wir erwähnten, unterziehen wir in diesem Zusammenhange die Zolltarife keiner weiteren Würdigung. Hingegen muß die Frachtenpolitik und die Stahlpreisgestaltung wegen ihrer großen Wichtigkeit kurz erwähnt werden. Beide laufen im Effekt darauf hinaus, dem nordöstlichen Industriegebiet seine beherrschende Stellung zu erhalten, und erschweren damit die industrielle Entwicklung des Südens und des Westens.

In welchem $\mathrm{Maße}$ die Bahnfrachten entscheidend wirken können, zeigt ein Vergleich der relativen Tarife in den fünf großen Tarifbezirken der USA. Sofern die Eastern Division mit 100 eingesetzt wird, ergeben sich für die andern Gebiete die folgenden Werte: Southern 139, Western Trunk Line 147, 
Southwestern 175 und Mountain Pacific 171. Uberall dort, wo Preisnotierungen f.o.b. stattfinden, wirken sich diese Differenzen stark aus. Zweifellos muß dieses ganze Problem, das den Süden und Westen in außerordentlichem $\mathrm{Maße}$ beschäftigt, auf einer höheren Plattform betrachtet werden als die, auf der sich unsere Ausführungen bewegen. Es handelt sich dabei um eine grundsätzliche Antithese zwischen Industrie- und Agrargebieten, wobei der Osten den Vorteil des frühen Starts und der entscheidenden Führung im Aufbau des amerikanischen Wirtschaftssystems besessen hatte.

Mit wenigen Ausnahmen werden die uns hier interessierenden Erzeugnisse jedoch nicht f.o.b. mill verkauft, sondern nach einem besonderen Preissystem, welches das "basing point system» genannt wird. Dabei handelt es sich im Prinzip un folgendes:

Unter qualitativer und quantitativer Spezifizierung sind für jedes in Frage kommende Produkt durch die Industrie auf den Basispunkt bezogene Preise festgesetzt worden. Sofern sich Produzent und Käufer beide am Basispunkt, z. B. in Pittsburgh, befinden, wird dem Käufer für die Lieferung der erwähnte Basispreis verrechnet; wohnt dagegen der Käufer an einem andem Orte als am Basispunkt, so wird zum Basispreis ein Zuschlag in der Höhe der publizierten Bahnfracht vom Basispunkt zu seinem Domizil hinzugeschlagen. Dieses "Plus» steht durchaus nicht immer in einer direkten Beziehung zu einer entsprechenden Arbeitsleistung. Es wird immer verrechnet, wenn der Käufer nicht am Basispunkt wohnt, und zwar gleichgültig, mit welchem Transportmittel der Transport erfolgt, ja sogar gleichgültig, wo der Lieferant wohnt. Das "Plus» wird beispielsweise auch berechnet, wenn Lieferant und Käufer an demselben Orte, der aber nicht Basispunkt ist, wohnen. Anderseits wird das "Plus" nicht verrechnet, wenn beispielsweise der Lieferant außerhalb des Basispunktes, der Käufer dagegen an demselben wohnt. So viel zur Erklärung des Basispunktsystems.

Seit Ende des 19. Jahrhunderts bis 1924 galt für Stahl als einziger Basispunkt Pittsburgh; der Zuschlag wurde als Pittsburgh-Plus bezeichnet. Mit dieser Maßnahme wurde selbstverständlich Pittsburgh als Industriezentrum weitgehend vor Dezentralisierungstendenzen geschützt. Nachdem im Jahre 1924 die Federal Trade Commission die US Steel Corporation zur Aufgabe des bisherigen Systems veranlaßt hatte, wurde nicht etwa das "basing point system" als solches aufgegeben, sondern ganz im Gegenteil noch ausgebaut. Es erstreckt sich heute auf fast alle Erzeugnisse der Eisen- und Stahlindustrie; nur ist an Stelle eines einzigen Basispunktes eine größere Zahl getreten, so daß nach dem zweiten Weltkriege jedes größere Produktionszentrum zu einem Basispunkt mit Ergänzungsgebiet gewvorden ist. Diese Umstände wirken nun in ähnlicher, nur multipler Weise als das frühere Pittsburgh-Plus, nämlich im Sinne einer Konsolidierung bestehender Produktionszentren, an denen sich mit Vorteil Produzenten wie Käufer von Erzeugnissen der Eisen- und Stahlindustrie ballen. Für die Industrie als Ganzes wirkt sich das Basispunktsystem nicht ungünstig aus; vor allem wird es für die relativ hohe Stabilität der Eisen- und Stahlpreise verantwortlich gemacht.

Damit hätten wir die. wichtigsten Faktoren, die den Standort der amerikanischen Schwerindustrie bestimmen und auf die Veränderungen desselben Einfluß haben, kennengelernt. Mit 511500 Lohnverdienern und einer jährlichen Lohnsumme von 750 Millionen Dollar stand 1939 die Eisen- und Stahlindustrie an erster Stelle, gefolgt von der Automobilindustrie mit 400000 Lohnverdienern und einer Lohnsumme von 650 Millionen Dollar. Sie zeichnete die industriellen Schwerpunkte der amerikanischen Wirtschaft.

Während des zweiten Weltkrieges stiegen die erzeugten Mengen auf nie vorher erreichte Höhen mit 118189000 Tonnen Eisenerz (1942), 61007000 Tonnen Roheisen (1944), 89642000 Tonnen Rohstahl (1944) an; die Zahl der Lohnverdiener erreichte im Juni 1942 mit 760400 das Maximum. Um diese ungeheure Produktionssteigerung zu besprechen, ziehen wir den Begriff der Produktionskapazität, deren Ausnützung und Ausweitung oder Vermehrung im folgenden bei.

Tab. 3: Roheisen- und Rohstahlkapazität und -produktion in Millionen Short tons; Ausnutzung der vorhandenen Kapazität in \%.

Roheisen*

Kapazität Produktion Ausnutzung

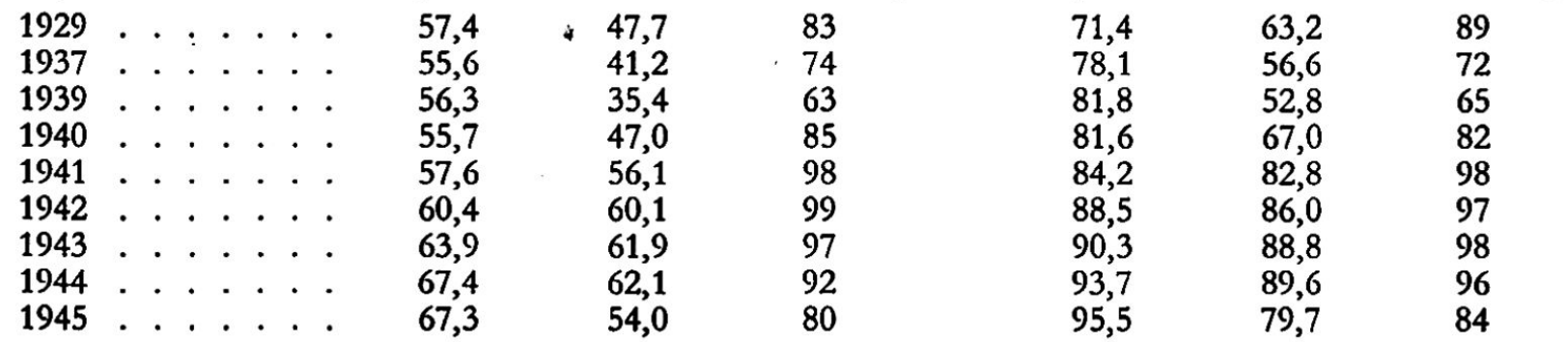

* Dic Angaben in Tabelle 3 für Roheisenproduktion sind etwas höher als die oben argegebenen Werte, da auch die im Hcchofen erzeugten Ferro alloys mitcingeschlossen sind. 
Grundlage unserer Betrachtung ist die Tabelle 3. Sie zeigt, daß in erster Linie die bessere Kapazitätsausnützung und erst in zweiter Linie die Kapazitätsvermehrung an der Produktionssteigerung schuld ist. Erwähnt werden muß auch, daß die durchschnittliche wöchentliche Arbeitszeit von 35,3 Stunden im Jahre 1939 sukzessive auf 46,3 Stunden im Jahre 1944 anstieg.

Eine durchgehende Kapazitätsausnützung zwischen 95 und $98 \%$ ist eine bewunderungswürdige Leistung, wenn man nur schon an die vielen Engpässe oder Flaschenhälse denkt. Zu diesen gehört auch eine während des Krieges ganz anders als im Frieden gerichtete Beanspruchung der einzelnen Produktionszweige, die schon in der zweiten Hälfte 1940 dazu führte, daß private und Regierungsaufträge in einzelnen Sektoren zur Erreichung der Kapazitätsgrenze führten, ja die vorhandene Leistungsfähigkeit überstiegen.

Dies führte dazu, daß im Oktober 1940 gesetzlich privaten Gesellschaften gestattet, wurde, neu errichtete Produktionsanlagen in dem kurzen Zeitraume von fünf Jahren abzuschreiben, sofern eine entsprechende Einwilligung des Office of Production Management vorlag. Damit wurde eine erste Etappe der Kapazitätsausweitung eingeleitet, die privat finanziert wurde und ohne generellen Richtplan erfolgt. Beispielsweise erhielt schon im November 1940 die Bethlehem Steel Corporation ein sogenanntes «certificate of necessity》 zur Erweiterung ihrer Rohstahlkapazität um 850000 Tonnen. Vom 1. Januar 1940 bis zum 1. Januar 1942 betrugen die Kapazitätsausweitungen nach diesem Plan:

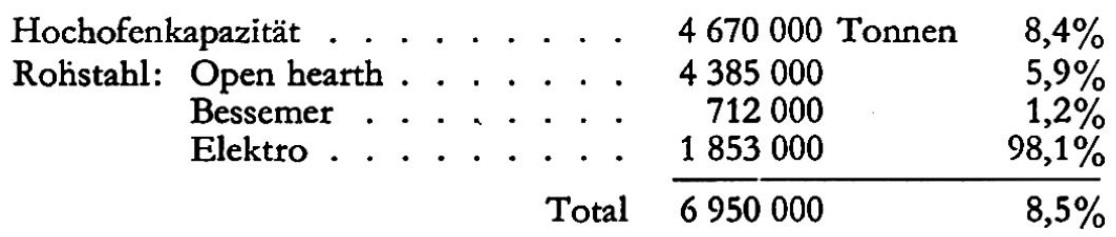

Zunehmende Stahlverknappung auf verschiedenen Sektoren und Gebieten (vor allem im Westen) führte dazu, daß Ende 1944 ein von der US-Regierung finanziertes und geplantes Erweiterungsprogramm in der Größenordnung von 10 Millionen Tonnen Stahl (über dem Werte vom 31. Mai 1941) in die Wege geleitet wurde. Diese zweite Etappe kam nie zur vollen Durchführung, indem durch verschiedene Korrekturen das Programm 1944 schließlich auf 7 Millionen Tonnen festgelegt wurde. Die Werte für die Zeit der zweiten Etappe vom 1. Januar 1942 bis zum 30. Juni 1945, die Prozentzahlen für die Zeit vom 1. Januar 1940 bis zum 30. Juni 1945 lauten:

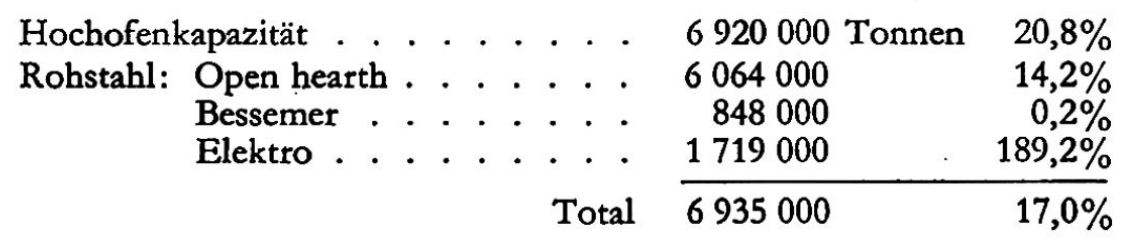

Die Kapazitätserweiterungen erfolgten entweder durch Errichtung neuer Anlagen unter neugebildeten Gesellschaften, durch Errichtung neuer Anlagen durch schon existierende Gesellschaften oder in den weitaus meisten Fällen durch die Erweiterung schon bestehender Produktionsanlagen. Als Beispiele der ersten Gruppe können dienen: die Kaiserwerke in Fontana, California, und Lone Star Blast Furnace in Daingerfield, Texas. In die zweite Gruppe gehören die von der US Steel betriebenen Hochöfen und Stahlwerke in Geneva, Utah, oder Sheffield Steel Co. in Houston, Texas. Die früher schon besonders hervorgehobenen drei größten Gesellschaften (US Steel, Bethlehem Steel und Republic Steel) waren mit 50,4\% an der Erweiterung der Hochofenkapazität und mit 53,1\% an derjenigen der Stahlwerke beteiligt. Rund 2,7 Milliarden Dollar verschlang das gesamte Erweiterungprogramm, wobei ziemlich genau die Hälfte, nämlich 1321 Millionen Dollar, auf Investierungen der US-Regierung entfiel. 
Für sich betrachtet, ist diese Ausweitung der Kapazität imponierend; es darf aber nicht übersehen werden, daß andere Industrien relativ zum Stande 1939 während des Krieges eine viel stärkere Entwicklung und Ausweitung erfuhren. Mit all den bisher besprochenen Maßnahmen wäre das Kriegsprogramm nicht zu erreichen gewesen, wenn nicht gleichzeitig auch eine äußerst kräftige Drosselung des zivilen Bedarfes stattgefunden hätte.

Tab. 4: Verteilung von «finished iron and steel products》 auf die verschiedenen Industriezweige in \% der USA

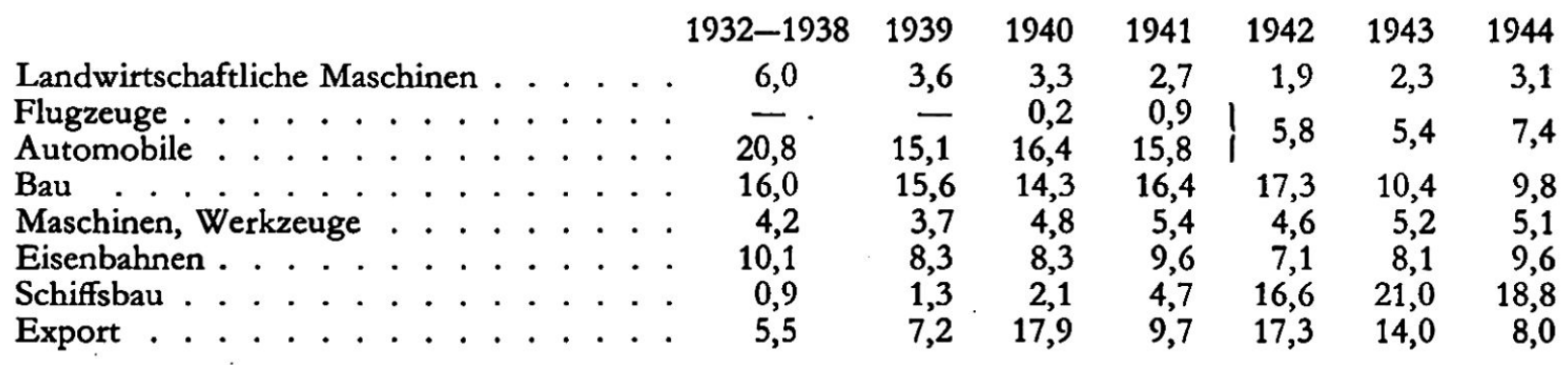

Die Feststellung, daß die nahe der Vorkriegsstahlbasis liegende Automobilindustrie von 20,8 \% Anteil 1932-1938 auf 5,4\% 1943 absank und anderseits Export und Schiffsbau zusammen von $6,4 \%$ auf $35 \%$ anstiegen, dürfte die Vermutung nahelegen, daß dies nicht ohne Einfluß auf die Standorte der neuerrichteten Werke bzw. Erweiterungen geblieben ist. Tabelle 5 zeigt uns dagegen ein ganz anderes Bild.

Tab. 5: Standorte der Stahlkapazität vor und nach dem zweiten Weltkriege

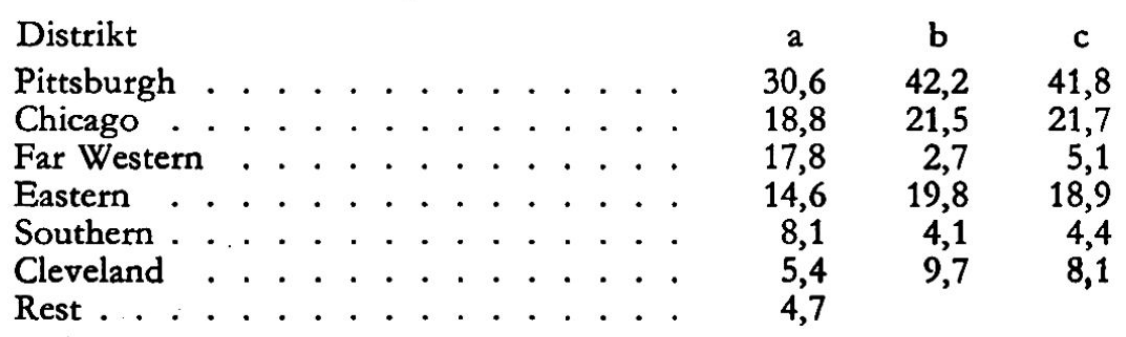

a: Prozentanteil an den Neuinvestierungen $1940-1945$.

b: Prozentanteil an der Stahlkapazität am 1. Januar 1940.

c: Prozentanteil an der Stahlkapazität am 1. Januar 1945.

Pittsburgh vermochte seine dominierende Stellung zu erhalten; der Mittelatlantische Distrikt nahm nicht etwa dank seiner günstigen Exportlage an Bedeutung zu, sondern im Gegenteil als Folge erschwerter Erzimporte relativ stark ab. Eine Verschiebung erfolgte vor allem gegen Westen und gegen Süden. Im großen blieb aber das Bild der allgemeinen Verteilung so, wie es vor dem Kriege war. Diese Feststellung erscheint bedeutsam, da eine Reihe von anderen Industrien außerordentlich starke Standortsänderungen während des Krieges, meist süd- oder westwärts, ausgeführt hat. Die imponierende Größe eines Hüttenwerkes, das wir im Westen neu erstehen sehen, beeindruckt uns so sehr, daß wir die Relation zum Ganzen leicht übersehen. Und im ganzen besehen, haben, wie die angegebenen Zahlenwerte es belegen, keine schwerindustriellen Standortsänderungen von Belang zwischen 1939 und 1945 stattgefunden.

Vom Standpunkte der Einzelregion aus gesehen, sind jedoch die neuen Schwerindustrien im Westen von brennendem Interesse. Hauptgrund zu ihrer Errichtung war zweifellos die mangelnde schwerindustrielle Basis des pazifischen Kriegstheaters. Die beiden wichtigsten neuen Anlagen befinden sich in Geneva, Utah, wo Eisenerz wie Kokskohle in nächster Nähe vorkommen, und in Fontana, California - unweit Los Angeles -, wo Eisenerz und Schrott vorhanden sind, während Kokskohle von Utah oder von noch weiter her per Achse zugeführt werden muß. Die von einer Tochtergesellschaft der US Steel betriebenen Werke in Utah besitzen eine Kapazität von 1238000 Tonnen Stahl. Die Anlage von Fontana besitzt eine Kapazität von 750000 Tonnen. Zusammen mit anderen, hier nicht namentlich 
erwähnten Werken kommt damit heute die Gesamtkapazität im Westen höher zu liegen als der zu erwartende Normalkonsum. Die Einfügung dieser großen kriegsbedingten Werke in die Friedensproduktion stellt manche Probleme: Es wurde beispielsweise berechnet, daß allein technische Umstellungen Neuinvestierungen von voraussichtlich 75 Millionen Dollar bedingen werden. Auf die Bedeutung der Differentialtarife, die freilich nach einem Entscheide der Interstate Commerce Commission vom Jahre 1945 sukzessive ausgeglichen werden sollen, wurde bei früherer Gelegenheit schon hingewiesen. Diese Angleichung der Frachttarife dürfte vor allem auch Birmingham eine vermehrte Möglichkeit geben, als Roheisenlieferant auf den nördlichen Märkten wieder in Erscheinung zu treten.

Nach dieser Darstellung der kriegśbedingten Veränderungen sei noch in Kürze ein Blick auf einige Probleme der Nachkriegszeit und der Zukunft geworfen.

Schon die Zahlen der Produktionskapazität und deren Ausnutzung zeigten nach Kriegsende ein leichtes Nachlassen der Periode höchster Anspannung. Die Produktionsspitze wurde bei Eisenerz schon 1942, bei Roheisen und Rohstahl 1944 erreicht; die Ausnutzung der Produktionskapazität zeigt erstmals 1944 fallende Tendenz. Selbst die Produktionskapazität ist stellenweise leicht rückläufig als Folge der Demontierung einzelner Anlagen. Als Ganzes jedoch ist die Schwerindustrie in erster Linie als Folge eines enormen Nachholbedarfes der für den Zivilbedarf arbeitenden Industrie andauernd vollbeschäftigt.

Auch die Exporte an Eisen und Stahl zeigen nach einem Maximum im Jahre 1940 andauernd sinkende Tendenz, wobei freilich einzelne Positionen, wie Eisenbahnschienen, Platten und Bleche, sich gegenteilig verhalten. Die Stellung der USA auf fremden Märkten war früher vor allem von seiten der europäischen Produzenten beeinträchtigt worden. Es ist in diesem Zusammenhange zu beachten, daß die meisten europäischen Länder das Vorkriegsniveau der Stahlproduktion erreicht, wenn nicht überschritten haben; nach 1950/51 dürfte deshalb Amerika erneut einer scharfen europäischen Konkurrenz ausgesetzt sein. Wie vor dem Kriege, befindet sich auch heute der Großteil der amerikanischen Schwerindustrie in ausgesprochener Binnenlage.

Damit im Zusammenhange wird das Problem der Preisbildung von größter Wichtigkeit, wobei wir hier lediglich auf die Rohstoffbeschaffung (Eisenerz und Koks) hinweisen wollen.

Wir erwähnten schon, daß in den USA praktisch alles Roheisen im mit Koks beschickten Blashochofen erzeugt wird. Diese Öfen verschluckten im Jahre 194548965000 Tonnen Koks oder $73 \%$ der US-Kokserzeugung. Koks ist ein wertvoller und Kokskohle relativ zu den gesamten Kohlevorräten rarer Artikel. Connellsville-Koks, ein Standarderzeugnis der pennsylvanischen Felder, zeigte beispielsweise folgende Preissteigerungen:

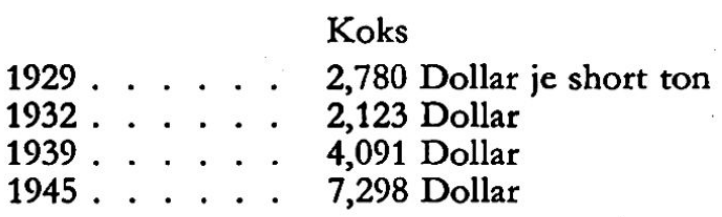

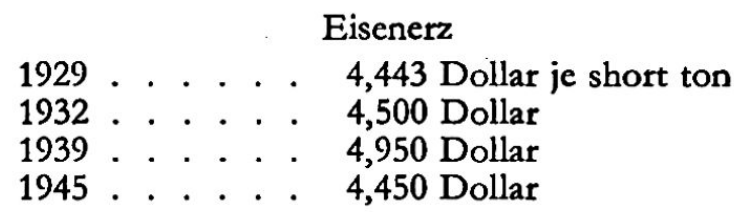

Es ist im Grunde erstaunlich, daß in den USA anscheinend neue Methoden der Roheisenerzeugung, die mit billigeren und reichlicher vorhandenen Kohlearten arbeiten, noch keinen ins Gewicht fallenden Eingang gefunden haben.

Besonders eigenartig jedoch sind die Verhältnisse mit Bezug auf die Erzbasis. Es wird viel zu wenig beachtet, daß fast die Hälfte der Weltschwerindustrie von einer Erzbasis beliefert wird, die etwa $1200 \mathrm{~km}$ vom Cleveland-Distrikt und $1750 \mathrm{~km}$ von Pittsburgh entfernt ist. Freilich ist zwischen diesen Punkten ein Transportapparat aufgebaut worden, der seinesgleichen auf der Erde nicht kennt. Äußerste Rationalisierung und Ausrichtung aller Transport- und Umladeeinrichtungen auf ein einziges Produkt, Eisenerz, gestatten, die "long ton" für $94 \mathrm{ct}$. über die Seen und für 3,25 Dollar von Lake Superior nach Pittsburgh zu befördern. Wie empfindlich diese Hauptarterie jedoch ist, mag ein Hinweis darauf zeigen, daß alle Transporte als Flaschenhals die Schleusenanlagen zwischen Lake Superior und Lake Huron am Soo-Kanal zu passieren haben. 
Häufig diskutiert ist auch die Lebensdauer der Erzreserven am Lake Superior. Die heute abgebauten hochwertigen Erze sind im Verhältnis zu den Abbaumengen nur in beschränktem $\mathrm{Maße}$ vorhanden. Festgestellte Reserven lassen eine Lebensdauer von etwa 10 Jahren (andere Schätzungen schwanken zwischen 5 und 25 Jahren) annehmen. Mit andern Worten: Die Frage der zukünftigen Erzbasis ist außerordentlich akut.

Anderseits sind hochwertige Eisenerze an andern Stellen im Seenbezirk und in Labrador erschlossen worden, die alle - wenigstens nach dem Ausbau des St.-LawrenceWasserweges - transportgünstig mit Bezug auf den heutigen Standort der amerikanischen Schwerindustrie liegen. Und zudem sind ungeheure Reserven an geringerwertigen Eisenerzen, sogenannte Taconite mit $25-35 \% \mathrm{Fe}$, im Lake-Superior-Distrikt vorhanden, die für Hunderte von Jahren die Erzversorgung zu sichern vermögen. Diese Taconite müßten aber an Ort und Stelle durch magnetische Trennung und Sintern angereichert werden, was umfangreiche Neuinvestierungen (die auf 1 Milliarde Dollar geschätzt werden) notwendig macht. Nach Berechnungen aus dem Jahre 1944 würden solche Konzentrate mit einem Fe-Gehalt von $62-65 \%$ 8,80 Dollar per Tonne Roheisen im Lake-Erie-Distrikt kosten, während im Durchschnitt der jetzt üblichen Lieferungen die Belastung 7,11 Dollar ausmacht. Wenn auch die Frage der zukünftigen Erzbasis die amerikanische Industrie sehr beschäftigt, so muß es doch als eine starke Verzerrung der tatsächlichen Verhältnisse bezeichnet werden, wenn gelegentlich von der baldigen Erschöpfung der Lake-Superior-Erze gesprochen wird.

Literaturhinweise (in erster Linie wurde für diese Arbeit Lit. 2 herbeigezogen): 1. Statistical Abstract of the United States 1947. Washington. - 2. United States Tariff Commission: Iron and Steel War Changes in Industry Series. Report No. 15. Washington 1946. - 3. Bureau of Mines and Geological Survey: Mineral Resources of the United States. Washington 1948. - 4. ANDERson, S. A., and Augustus, Jones: Iron in the Adirondacks. Economic Geography 21, 1945, 276-285. - 5. AppLETON, B.: Iron and Steel Industry of the Cleveland District. Economic Geography 5, 1929, 308-319. 6. BoEsCH, H.: Birmingham (Jefferson County), Alabama (USA); Wirtschaftsgeographie eines Schwerindustriegebietes. Vierteljahresschrift der Naturforschenden Gesellschaft Zürich, 1945. - 7. BRIGHTMAN, G. F.: Cuyuna Iron Range. Economic Geography 18, 1942, 275-286. - 8. CarLSON, A. S., and Charles B. Gow: Scrap Iron and Steel Industry. Economic Geography 12, 1936, 175-184. - 9. CreDNER, W.: Die Eisenerzgrundlagen der Vereinigten Staaten von Amerika. Lebensraumfragen, III, 1943, 701-726. - 10. Dietrich, B.: Die Verlagerung der Industrien in den Vereinigten Staaten von Amerika. Lebensraumfragen, III, 1943, 727-774. - 11. JoNEs, C. F.: Areal Distribution of Manufacturing in the United States. Economic Geography 14, 1938, 217-222. - 12. National Resources Planning Board: Industrial Location and National Resources. Washington 1943. - 13. Perrson, E. W.: The Mineral Position of the United States and the Outlook for the Future. Smithsonian Report 1945. 14. Primmer, G. H.: Future of Lake Superior Ore Supply. Economic Geography 10, 1934, 395-401. 15. RENNER, G.: Geography of Industrial Localization. Economic Geography 23, 1947, 167-189. 16. White, L.: Geography's Part in the Plant Cost of Iron and Steel Production at Pittsburgh, Chicago and Birmingham. Economic Geography 5, 1929, 327-334.

\section{L'INDUSTRIE LOURDE AMERICAINE}

A l'aide de la littérature et de la statistique, on enquête en quelle mesure, pendant la seconde guerre mondiale, l'industrie lourde américaine a subi des changements d'emplacements. Bien que les usines établies à nouveau dans le sud et l'ouest du pays sont de la plus grande importance pour la région en question, elles n'ont pas pu disloquer le centre d'attraction d'industries lourde qui se trouve dans la région de Pittsburgh-Cleveland-Chicago. Avec une participation à la production mondiale de fer et d'acier bruts d'environ $40 \%$ la région est restée le plus important centre de l'industrie lourde du monde.

\section{L'INDUSTRIA PESANTE AMERICANA}

Sulla scorta della letteratura e delle statistiche si cerca di stabilire in quale grado l'industria pesante americana ha subito uno spostamento spaziale durante la seconda guerra mondiale. Benchè i nuovi centri industriali sorti nel sud e nell'ovest siano di capitale importanza per le corrispondenti zone, essi non riuscirono a spostare il centro di gravità dalla zona Pittsburgh-Cleveland-Chicago, la quale continua cosi a rimanere il centro dell'industria pesante più importante di tutto il mondo con una produzione di ferro e di acciaio greggio che rappresenta il $40 \%$ di quella mondiale. 